\title{
Tunable Optoelectronic Oscillator Incorporating a Single Passband Microwave Photonic Filter
}

\author{
Jiejun Zhang, Student Member, IEEE, Liang Gao, Student Member, IEEE, and Jianping Yao, Fellow, IEEE
}

\begin{abstract}
A wideband and continuously tunable optoelectronic oscillator (OEO) incorporating a single passband microwave photonic filter (MPF) is proposed and experimentally demonstrated. The single passband MPF is implemented using a broadband light source, a Mach-Zehnder interferometer (MZI) in which a phase modulator is incorporated in one arm, a dispersion compensating fiber (DCF), and a photodetector (PD). The light wave from the broadband light source is sent to the MZI, and the output signal from the MZI is sent to the DCF and then detected at the PD. The overall operation is equivalent to a single passband MPF with the central frequency of the passband tunable by tuning the length difference between the two arms of the MZI. By incorporating the MPF into an OEO, a microwave signal with a tunable frequency is generated. The proposed OEO is experimentally evaluated. A microwave signal with a tunable frequency from 1 to $12 \mathrm{GHz}$ and a tuning step of $0.5 \mathrm{GHz}$ is generated. The phase noise performance of the generated microwave signal is evaluated. At $6.02 \mathrm{GHz}$, the phase noise is measured to be $-107 \mathrm{dBc} / \mathrm{Hz}$ at an offset of $10 \mathrm{kHz}$.
\end{abstract}

Index Terms-Microwave generation, microwave photonics, optoelectronic oscillator.

\section{INTRODUCTION}

$\mathbf{T}$ HE GENERATION of a wideband and frequency tunable microwave signal is important for many applications, such as wireless communications, optical signal processing, radar, warfare systems, and modern instrumentation [1]. An optoelectronic oscillator (OEO) is considered one of the most effective solutions for the generation of a microwave signal with a low phase noise [2]-[11]. In an OEO, to ensure a single frequency operation, a narrowband electrical filter is usually employed in the loop [2]-[5]. To achieve frequency tuning, however, the microwave filter in an OEO must be tunable, which is hard to implement, especially for an OEO with a large frequency tunable range. For example, in [5] a tunable OEO was demonstrated in which the tuning was realized by changing the dispersion of the OEO loop through tuning the optical wavelength. Due to the use of a fixed electrical filter for frequency selection, the tunable range is very limited. In [6], an OEO based on cascaded circulating delay line was proposed. Frequency tuning of less than $3 \mathrm{GHz}$

Manuscript received June 21, 2013; revised November 13, 2013; accepted November 27, 2013. Date of publication December 3, 2013; date of current version January 21, 2014. This work was supported by the Natural Sciences and Engineering Research Council of Canada. The work of J. Zhang and L. Gao was supported by the China Scholarship Council.

The authors are with the Microwave Photonics Research Laboratory, School of Electrical Engineering and Computer Science, University of Ottawa, Ottawa, ON K1N 6N5, Canada (e-mail: jpyao@eecs.uottawa.ca).

Color versions of one or more of the figures in this letter are available online at http://ieeexplore.ieee.org.

Digital Object Identifier 10.1109/LPT.2013.2293505 was realized by changing the lengths of the delay lines. To implement an OEO with a large frequency tunable range, a new solution to implement a frequency tunable microwave photonic filter (MPF) based on phase modulation and phase-modulation to intensity-modulation (PM-IM) conversion in a chirped fiber Bragg grating (CFBG) was demonstrated [7]. The joint operation of the phase modulator (PM) and the CFBG corresponds to a MPF with an optically tunable central frequency. By incorporating the MPF into an OEO loop, a frequency tunable OEO was implemented in which the frequency tuning was realized by tuning the dispersion of the CFBG. A frequency tunable range of $6 \mathrm{GHz}$ was demonstrated. To ensure a large frequency tunable range, the value of dispersion must be tuned in a large range, which is hard to realize for a practical system. Recently, an OEO employing a PM and a phase-shifted fiber Bragg grating (PS-FBG) with a larger frequency tunable range was proposed and demonstrated [8]. Again, the joint operation of the PM and the PS-FBG corresponds to an MPF with the central frequency tunable by simply tuning the optical wavelength. An OEO with a tunable range over $10 \mathrm{GHz}$ was demonstrated [8]. A similar OEO based on PS-FBG with a tuning range of 3.8 $\mathrm{GHz}$ was demonstrated in [9]. The key limitation of the tunable OEO in [8], [9] is that the microwave frequency is directly determined by the wavelength spacing between the wavelength of the tunable laser source (TLS) and the center wavelength of the notch in the PS-FBG. Thus, a small drift of the wavelength of the TLS or the notch in the PS-FBG would lead to a large drift of the microwave frequency. Recently, an OEO with a large frequency tuning range of $34 \mathrm{GHz}$ was demonstrated [10]. The tuning was implemented by tuning the center wavelength of an optical bandpass filter. The major limitation of the approach is the optical bandpass filter must have an ultra-narrow bandwidth to ensure a single frequency operation, which is extremely difficult to realize, especially for a tunable optical filter.

More recently, a frequency-tunable OEO based on a microwave photonic finite impulse response (FIR) filter with a large frequency tunable range was demonstrated [11]. The filter was implemented using a broadband light source sliced by an optical comb filter with the time delays introduced by a dispersive fiber. By incorporating the microwave photonic FIR filter into an OEO loop, a frequency tunable OEO was realized. The major limitation of this approach is that the tuning of the FIR filter was realized by changing the comb spacing of the optical comb filter, which is again complicated and costly. In addition, due to the periodic nature of the spectral response of the FIR filter, the tunable range is limited 


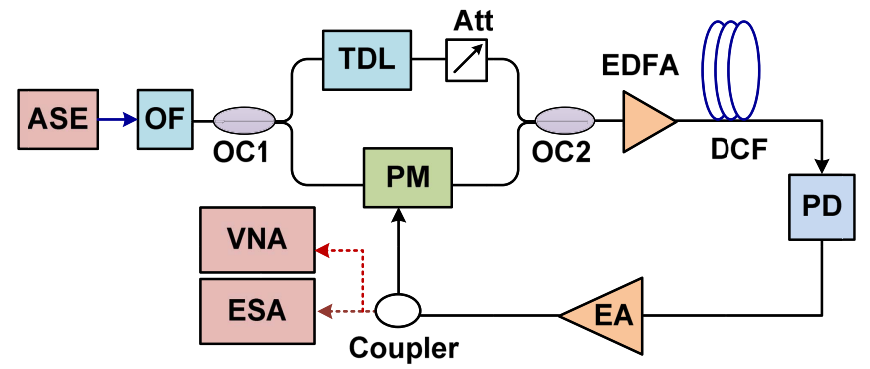

Fig. 1. Schematic of the proposed OEO. ASE: amplified spontaneous emission, OF; optical filter, TDL: tunable delay line, PM: phase modulator, Att: attenuator, OC: optical coupler, EDFA: erbium-doped fiber amplifier, DCF: dispersion compensating fiber, PD: photodetector, EA: electrical amplifier, VNA: vector network analyzer, ESA: electrical spectrum analyzer.

by the free spectral range (FSR). Single passband MPF with wideband tunable range was proposed by Mora et al. in [12] by using a broadband optical source that is spliced by a Mach-Zehnder interferometer (MZI). Since only a single passband is generated, the use of the filter will increase the tunable range.

In this letter, we propose a wideband and continuously frequency-tunable OEO based on a single passband MPF. The MPF is implemented using a broadband light source that is sent to a MZI in which a PM is incorporated in one arm. The optical signal at the output of the MZI is sent to a dispersive device which is a length of dispersion compensating fiber (DCF) in our demonstration, and then detected at a photodetector (PD). The overall operation of the system is equivalent to a single passband MPF with the central frequency of the passband tunable by tuning the length difference between the two arms of the MZI. By incorporating the MPF into an OEO, a frequency-tunable microwave signal is generated. An experiment is performed. A microwave signal with a tunable frequency from 1 to $12 \mathrm{GHz}$ and a tuning step of $0.5 \mathrm{GHz}$ is generated. The phase noise performance of the generated microwave signal is also evaluated. At $6.02 \mathrm{GHz}$, the phase noise is measured to be $-107 \mathrm{dBc} / \mathrm{Hz}$ at an offset of $10 \mathrm{kHz}$.

\section{PRINCIPLE}

Fig. 1 shows the schematic of the proposed OEO. The spectrum of an amplified spontaneous emission (ASE) source is flattened by an optical filter (OF) and then coupled into an MZI through an optical coupler (OC1). In the upper arm of the MZI, a tunable optical delay line (TDL) is used to change the length difference of the MZI and a tunable attenuator (Att) is used to balance the optical powers between the two channels. A PM to which a generated microwave signal is applied is incorporated into the lower arm of the MZI. After passing through the MZI, the optical signal is amplified by an erbium-doped fiber amplifier (EDFA), and sent to a PD through a length of DCF and then detected at a PD. The electrical signal at the output of the PD is amplified by an electrical amplifier (EA) and applied to the PM, to close the OEO loop.

In the following, the operation of the OEO is discussed. First, the open-loop frequency response of the OEO is analyzed. Assume that the length difference between the two arms of the MZI is $\Delta l$, the electrical field at the output of the upper arm of the MZI is given by $E_{1}=E_{0} e^{j k \Delta l}$, where $k$ is the wave-number and $E_{0}$ is the amplitude of the electrical filed at the input of the upper arm. For the lower arm, the electrical field at the output of the PM is given by $E_{2}=E_{0} e^{j \varphi(t)}$, where $\varphi(t)=\gamma_{P M} f(t)$ is the phase change which is introduced by the modulating signal $f(t)$, and $\gamma_{P M}$ is the phase modulation index [13]. $E_{1}$ and $E_{2}$ are then combined at a second optical coupler (OC2), the electrical field at the output of OC2 is given by

$$
E_{3}=E_{1}+E_{2}=E_{0} e^{j k \Delta l}+E_{0} e^{j \varphi(t)}
$$

The DCF would introduce an optical-frequency-dependent time delay. The electrical field at the output of the DCF is given by

$$
E_{4}=\left.E_{3}\right|_{t=t_{0}-\tau_{0}-L \beta \Delta \omega}=E_{0} e^{j \omega n \Delta l / c}+E_{0} e^{j \varphi\left(t_{0}-L \beta \omega\right)}
$$

where $L$ and $\beta$ are the length and the group velocity dispersion parameter of the DCF, $\omega$ is the angular frequency of the optical carrier, $c$ is light speed in vacuum, $n$ is the refractive index of the optical fiber, $t_{0}$ represents the initial time delay of the modulation signal, and $\tau_{0}$ is the time delay caused by the length of the DCF. Since $t_{0}$ and $\tau_{0}$ are two constants, for simplicity, they can be ignored. The optical power at the input of the PD can be calculated by

$$
\begin{aligned}
P & =\int_{\delta \omega} E_{4} \cdot E_{4}^{*} d \omega \\
& =\int_{\delta \omega}\left[2 E_{0}^{2}+2 E_{0}^{2} \cos [\omega n \Delta l / c-\varphi(-L \beta \omega)]\right] d \omega
\end{aligned}
$$

where $\delta \omega$ is the optical bandwidth of the ASE source. The first term in (3) is a DC term and can be ignored. When a modulation signal of $f(t)=A \cos (\Omega t)$ is applied, where $A$ and $\Omega$ are the amplitude and angular frequency of the modulation signal, a photo-current at the output of the PD is generated, which is given by

$$
\begin{aligned}
i(\Omega)= & 2 R \times E_{0}^{2} \int_{\delta \omega} \cos \left[\omega n \Delta l / c-A \gamma_{P M} \cos (-\Omega L \beta \omega)\right] d \omega \\
= & 2 R \times E_{0}^{2} \operatorname{Re}\left[\int_{\delta \omega} e^{j \omega n \Delta l / c-j A \gamma_{P M} \cos (-\Omega L \beta \omega)} d \omega\right] \\
\approx & 2 R \times E_{0}^{2} \operatorname{Re}\left\{\int _ { \delta \omega } e ^ { j \omega n \Delta l / c } \left[J_{0}\left(A_{P M}\right)\right.\right. \\
& \left.\left.+J_{1}\left(A \gamma_{P M}\right) e^{-j \Omega L \beta \omega}+J_{-1}\left(A_{P M}\right) e^{j \Omega L \beta \omega}\right] d \omega\right\}
\end{aligned}
$$

where $R$ is the responsivity of the PD, and $J_{n}\left(A \gamma_{P M}\right)$ is the $n$-th Bessel function of the first kind. Again, the constant phase terms and the DC term $J_{0}\left(A \gamma_{P M}\right)$ can be ignored, the 
photo-current can then be further written as

$$
\begin{aligned}
i(\Omega)= & 2 R \times E_{0}^{2} J_{1}\left(A \gamma_{P M}\right) \cdot \int_{\delta \omega} \cos [(n \Delta l / c-\Omega L \beta) \omega] d \omega \\
& -2 R \times E_{0}^{2} J_{1}\left(A \gamma_{P M}\right) \cdot \int_{\delta \omega} \cos [(n \Delta l / c+\Omega L \beta) \omega] d \omega \\
= & R \times P_{A} J_{1}\left(A \gamma_{P M}\right) \operatorname{sinc}[(n \Delta l / c-\Omega L \beta) \delta \omega] \\
& -R \times P_{A} J_{1}\left(A \gamma_{P M}\right) \operatorname{sinc}[(n \Delta l / c+\Omega L \beta) \delta \omega]
\end{aligned}
$$

where $P_{A}=2 E_{0}^{2} \delta \omega$ is the optical power of the ASE source. Considering the input microwave signal is $f(t)=A \cos (\Omega t)$, the transfer function of the open loop, corresponding to a single passband MPF, can be written as $H(\Omega)=i(\Omega) / A$.

As can be seen from (5), the transfer function of the single passband MPF is in the form of a sinc function, thus the MPF has only a single passband at a frequency where $\left(n \Delta l / c \pm \Omega_{c} L \beta\right) \delta \omega=0$ or $\Omega_{c}=n \Delta l /(c L \beta)$. The center frequency of the passband of the MPF is then given by

$$
f=\frac{\Omega_{c}}{2 \pi}=\frac{n \Delta l}{2 \pi c L \beta}=\frac{1}{L \beta \omega_{F S R}}
$$

where $\omega_{F S R}=2 \pi c /(n \Delta l)$ is the FSR of the MZI in angular frequency. As can be seen, the central frequency of the MPF is proportional to the length difference between the two arms of the MZI, thus the MPF can be tuned by tuning the length of the TDL.

Then, the bandwidth of the MPF is evaluated. For a sinc function, the frequencies $\Omega_{ \pm 1}$ of the nearest zeros to $\Omega_{c}$ can be achieved by letting $\left(n \Delta l / c-\Omega_{ \pm 1} L \beta\right) \delta \omega= \pm \pi$

$$
\Omega_{ \pm 1}=\frac{n \Delta l / c \pm \pi / \delta \omega}{L \beta}
$$

And the null-to-null bandwidth of the filter is then given

$$
\Delta \Omega_{ \pm 1}=2\left|\Omega_{c}-\Omega_{+1}\right|=\frac{2 \pi}{L \beta \delta \omega}
$$

It can be seen that the bandwidth of the MPF is inversely proportional to the spectral width of the optical source and the total dispersion of the DCF. Hence, by using an optical source with a wider spectral range and a DCF with a larger dispersion value, the bandwidth of the single passband MPF can be smaller. If the bandwidth is sufficiently small, only one mode will be supported and a single frequency oscillation is thus ensured. In addition, since no dispersion-induced power penalty term appears in (5), the generation of a microwave signal at any frequency is enabled [14]. By incorporating the frequency-tunable single passband MPF in an OEO, a single frequency oscillation with an ultra-wide frequency tunable range can be achieved.

\section{EXPERIMENT}

An experiment based on the setup shown in Fig. 1 is performed. An ASE source with a $10-\mathrm{dB}$ spectral width of $30 \mathrm{~nm}$ is used as the broadband optical source. A reconfigurable optical filter (Finisar WaveShaper 4000S) is used to flatten the ASE spectrum and pre-compensate for the non-flat gain spectrum of the EDFA. The dispersion parameter of the DCF is $432 \mathrm{ps}^{2}$. The maximum time delay introduced

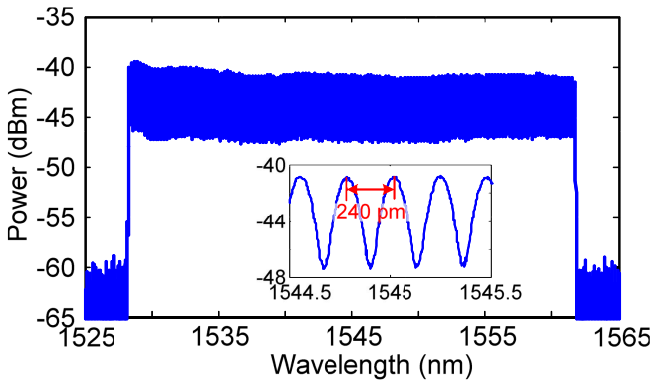

Fig. 2. Optical spectrum measured at the input of the PD. Inset: zoomed-in view of the optical spectrum from 1544.5 to $1545.5 \mathrm{~nm}$.

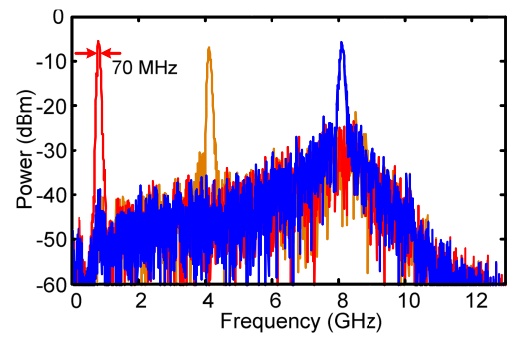

Fig. 3. Frequency response of the single passband MWF measured by a VNA. The central frequencies of the passband at 0.80 (red), 4.12 (yellow) and $8.10 \mathrm{GHz}$ (blue) by tuning the TDL are shown.

by the TDL is $660 \mathrm{ps}$, corresponding to the time delay of a 13.4-cm single-mode fiber. According to (6), the theoretical frequency tunable range of the MPF is $87.1 \mathrm{GHz}$, which is not achievable in our experiment due to the limited bandwidths of the PM, the PD and the EA. In the experimental setup, the operation bandwidths of the PM, the PD and the EA can cover a frequency range from 1 to $12 \mathrm{GHz}$.

Fig. 2 shows the optical spectrum from 1525 to $1565 \mathrm{~nm}$ measured at the input of the PD, which has a sinusoidal shape with a FSR of $240 \mathrm{pm}$. A zoom-in view of the spectrum is shown as an inset in Fig. 2. The FSR of the MZI can be continuously tuned by tuning the TDL in the upper arm of the MZI. By adjusting the optical attenuator in the upper arm, an extinction ratio of $6 \mathrm{~dB}$ of the spectrum is achieved.

Then, the open-loop response of the OEO corresponding to a single passband MPF is measured using a vector network analyzer (VNA, Agilent E8364A). Fig. 3 shows the measured MPF response when the passband frequency is located at $0.80 \mathrm{GHz}, 4.12 \mathrm{GHz}$ and $8.10 \mathrm{GHz}$ by tuning the TDL. The 3-dB bandwidth of the passband is around $70 \mathrm{MHz}$, which is greater than the theoretical value of $30 \mathrm{MHz}$ calculated by (8) due to the existence of high order dispersion in the DCF. Note that the MPF has a relatively high noise floor, which is resulted mainly from the noise terms from the ASE, the EDFA, and the PD.

By controlling the power of the EDFA and the gain of the EA to make OEO net loop gain greater than $0 \mathrm{~dB}$, the $\mathrm{OEO}$ will start to oscillate. The tuning of the microwave frequency is realized by tuning the TDL. Fig. 4 shows the superimposed spectra of the generated microwave signal, which is tuned over a frequency range from 1 to $12 \mathrm{GHz}$ with a tuning step of $0.5 \mathrm{GHz}$. The spectra are measured by an electrical spectrum 


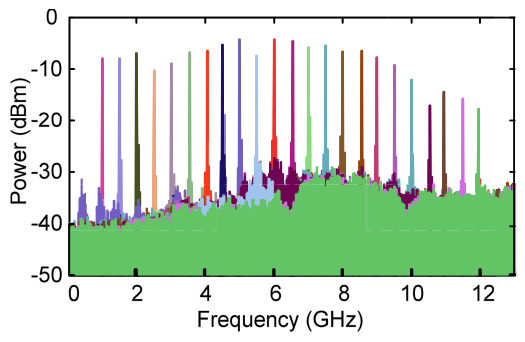

Fig. 4. Spectra of the generated microwave signal at different frequencies. The frequency is tuned from 1 to $12 \mathrm{GHz}$ with a tuning step of $0.5 \mathrm{GHz}$.
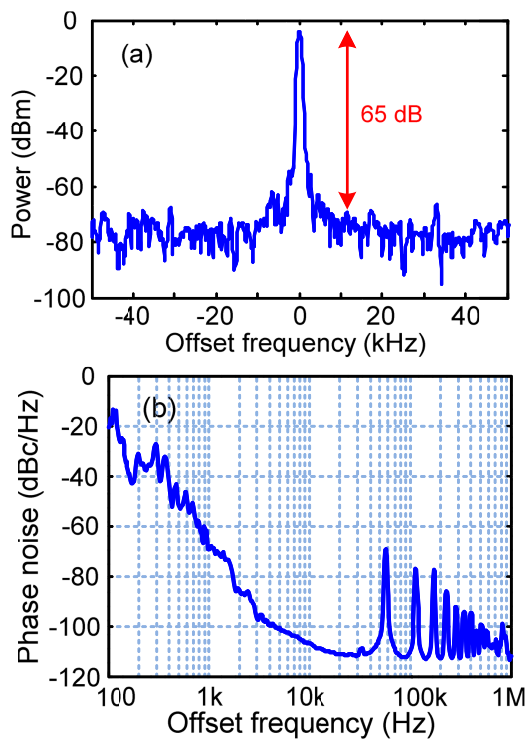

Fig. 5. (a) The zoomed-in view of the microwave signal at $6.02 \mathrm{GHz}$. (b) Measured phase noise spectrum of the $6.02 \mathrm{GHz}$ microwave signal.

analyzer (Agilent E4448A). The power variation during the tuning is resulted from the uneven frequency response of the electrical and the optical devices in the system. The signal to noise ratio (SNR) is measured to be over $30 \mathrm{~dB}$ over the entire tunable range, which can be further increased if a low-noise EDFA is employed.

Fig. 5(a) shows a zoom-in view of the microwave signal generated at $6.02 \mathrm{GHz}$, indicating a SNR of $65 \mathrm{~dB}$. The phase noise performance of the generated microwave signal is evaluated, which is done using an Agilent E5052B signal source analyzer incorporating an Agilent E5053A downconverter. From Fig. 5(b) we can see that the phase noise of the generated microwave signal is $-107 \mathrm{dBc} / \mathrm{Hz}$ at an offset frequency of $10 \mathrm{kHz}$. Several peaks for an offset frequency greater than $50 \mathrm{kHz}$ are observed, which are resulted from the sidemodes of the OEO. The first peak is located at $57.5-\mathrm{kHz}$ offset frequency, which indicates that the length of the OEO loop is $3.53 \mathrm{~km}$.

\section{CONCLUSION}

The phase noise of the OEO was measured to be $-107 \mathrm{dBc} / \mathrm{Hz}$ at an offset frequency of $10 \mathrm{kHz}$, which can be improved to be better than $-130 \mathrm{dBc} / \mathrm{Hz}$ by reducing the loss of the loop and decreasing the loop noise using amplifiers with lower noise figures. The use of a PM with smaller halfwave voltage, a PD with a higher responsivity could also improve the phase noise performance. The use of an OEO loop with a longer loop length can also improve the phase noise performance.

In conclusion, an ultra-wideband continuously frequencytunable OEO was proposed and experimentally demonstrated. The large frequency tunable range was ensured due to the use of a tunable single-passband MPF, which was implemented using a broadband light source, a MZI incorporating a PM in one arm, a DCF and a PD. The overall operation was equivalent to a single passband MPF with the central frequency of the passband tunable by tuning the length difference between the two arms of the MZI. An experiment was performed. A microwave signal with a frequency tunable from 1 to $12 \mathrm{GHz}$ was generated. The tuning range of the proposed OEO can be as large as $87.1 \mathrm{GHz}$. In the experiment, the tunable range was limited to $1-12 \mathrm{GHz}$, due to the limited bandwidths of the PM, the PD and the EA.

\section{REFERENCES}

[1] J. P. Yao, "Microwave photonics," J. Lightw. Technol., vol. 27, no. 3, pp. 314-335, Feb. 1, 2009.

[2] X. S. Yao and L. Maleki, "Optoelectronic microwave oscillator," J. Opt. Soc. Amer. B, vol. 13, no. 8, pp. 1725-1735, Aug. 1996.

[3] E. Shumakher, S. Dúill, and G. Eisenstein, "Optoelectronic oscillator tunable by an SOA based slow light element," J. Lightw. Technol., vol. 27, no. 18, pp. 4063-4068, Sep. 15, 2009.

[4] S. Pan and J. P. Yao, "A frequency-doubling optoelectronic oscillator using a polarization modulator," IEEE Photon. Technol. Lett., vol. 21, no. 13, pp. 929-931, Jul. 1, 2009.

[5] S. Poinsot, H. Porte, J. Goedgebuer, W. Rhodes, and B. Boussert, "Continuous radio-frequency tuning of an optoelectronic oscillator with dispersive feedback," Opt. Lett., vol. 27, no. 15, pp. 1300-1302, Aug. 2002.

[6] X. Liu, W. Pan, X. Zou, B. Luo, L. Yan, and B. Lu, "A reconfigurable optoelectronic oscillator based on cascaded coherence-controllable recirculating delay lines," Opt. Express, vol. 20, no. 12, pp. 13296-13301, Jun. 2012.

[7] W. Li and J. P. Yao, "An optically tunable optoelectronic oscillator," J. Lightw. Technol., vol. 28, no. 18, pp. 2640-2645, Sep. 15, 2010.

[8] W. Li and J. P. Yao, "A wideband frequency-tunable optoelectronic oscillator incorporating a tunable microwave-photonic filter based on phase-modulation to intensity-modulation conversion using a phaseshifted fiber Bragg grating," IEEE Trans. Microw. Theory Tech., vol. 60, no. 6, pp. 1735-1742, Jun. 2012.

[9] B. Yang, X. Jin, X. Zhang, S. Zheng, H. Chi, and Y. Wang, "A wideband frequency-tunable optoelectronic oscillator based on a narrowband phase-shifted FBG and wavelength tuning of laser," IEEE Photon. Technol. Lett., vol. 24, no. 1, pp. 73-75, Jan. 1, 2012.

[10] X. Xie, C. Zhang, T. Sun, P. Guo, X. Zhu, L. Zhu, et al., "Wideband tunable optoelectronic oscillator based on a phase modulator and a tunable optical filter," Opt. Lett., vol. 38, no. 5, pp. 655-657, Mar. 2013.

[11] M. Li, W. Li, and J. P. Yao, "Tunable optoelectronic oscillator incorporating a high-Q spectrum-sliced photonic microwave transversal filter," IEEE Photon. Technol. Lett., vol. 24, no. 14, pp. 1251-1253, Jul. 15, 2012.

[12] J. Mora, et al., "Photonic microwave tunable single-bandpass filter based on a Mach-Zehnder interferometer," J. Lightw. Technol., vol. 24, no. 7, pp. 2500-2509, Jul. 2006.

[13] J. P. Yao, F. Zeng, and Q. Wang, "Photonic generation of ultra-wideband signals," J. Lightw. Technol., vol. 25, no. 11, pp. 3219-3235, Nov. 2007.

[14] X. Xue, X. Zheng, H. Zhang, and B. Zhou, "Widely tunable singlebandpass microwave photonic filter employing a non-sliced broadband optical source," Opt. Express, vol. 19, no. 19, pp. 18423-18429, Sep. 2011. 